\title{
QCD matrix elements + parton showers. The NLO
}

\section{case}

\author{
Stefan Höche, ${ }^{a}$ Frank Krauss, ${ }^{b}$ Marek Schönherr ${ }^{b}$ and Frank Siegert ${ }^{c}$ \\ ${ }^{a}$ SLAC National Accelerator Laboratory, \\ Menlo Park, CA 94025, U.S.A. \\ ${ }^{b}$ Institute for Particle Physics Phenomenology, \\ Durham University, Durham DH1 3LE, U.K. \\ ${ }^{c}$ Physikalisches Institut, Albert-Ludwigs-Universität Freiburg, \\ D-79104 Freiburg, Germany \\ E-mail: shoeche@slac.stanford.edu, frank.krauss@durham.ac.uk, \\ marek.schoenherr@durham.ac.uk, frank.siegert@cern.ch
}

ABSTRACT: We present a process-independent technique to consistently combine nextto-leading order parton-level calculations of varying jet multiplicity and parton showers. Double counting is avoided by means of a modified truncated shower scheme. This method preserves both the fixed-order accuracy of the parton-level result and the logarithmic accuracy of the parton shower. We discuss the renormalisation and factorisation scale dependence of the approach and present results from an automated implementation in the SHERPA event generator using the test case of $W$-boson production at the Large Hadron Collider. We observe a dramatic reduction of theoretical uncertainties compared to existing methods which underlines the predictive power of our novel technique.

Keywords: Monte Carlo Simulations, NLO Computations

ARXIV EPRINT: 1207.5030 


\section{Contents}

1 Introduction 1

2 Multijet merging at next-to leading order - MEPs@NLO 2

2.1 Ingredients of the merging method 3

2.2 Definition of the merging method 6

$\begin{array}{lll}2.3 & \text { Fixed-order and logarithmic accuracy } & 10\end{array}$

$\begin{array}{lll}2.4 & \text { Renormalisation and factorisation scale uncertainties } & 11\end{array}$

3 Monte-Carlo implementation 12

$\begin{array}{lll}4 & \text { Results } & 13\end{array}$

$\begin{array}{llr}5 & \text { Conclusions } & 16\end{array}$

\section{Introduction}

Events with large final-state multiplicity, and in particular with many jets, are notorious in recent and present particle physics experiments at the energy frontier. This is especially true for measurements at the Large Hadron Collider, which operates at the highest centre-of-mass energies ever achieved in an accelerator-based experiment. Multijet events constitute a significant fraction of both signals and backgrounds. They must therefore be described with a theoretical precision that aims at matching the experimental one, or better.

Triggered by this necessity, astounding practical improvements in event simulation have occurred over the past decade, with far-reaching consequences for particle physics phenomenology, including precision physics and searches for new phenomena alike. These developments range from the (near) automation of cross section calculations for multiparticle final states at the next-to leading order [1-16] to the construction of algorithms that allow to use multi-particle calculations at leading and next-to-leading order to drive event simulation at the particle level.

For the latter point, it is necessary to consistently combine fixed-order matrix elements with parton showers. Two alternative ideas have been pursued in this regard: An exact matching of next-to-leading order calculations to parton showers has been worked out in the MC@NLo [17-22] and Powheg [23-28] approaches. For the latter, first steps to also incorporate electroweak corrections have been reported in [29, 30]. In general, both these methods are suitable for simulating inclusive processes, but they fail to describe the precise kinematics of multi-jet final states as part of the inclusive reaction. Alternatively, algorithms to merge various leading-order matrix elements with increasing parton multiplicity 
into one inclusive sample have been proposed [31-37]. They describe the inclusive cross section at Born level only, but each additional jet is also described by exact leading-order matrix elements, while respecting the overall logarithmic accuracy of the parton shower. This is best achieved by implementing truncated vetoed parton evolution [35, 36]. A combination of MC@NLO and MEPS, called MENLOPS, was recently introduced [38, 39], which allows to promote the cross section of the inclusive MEPS sample to NLO accuracy.

In this publication we propose a new method to merge next-to leading order predictions for the production of multi-jet final states in hadron-hadron collisions with the parton shower, maintaining both the fixed order accuracy for each jet multiplicity and the logarithmic accuracy of the parton shower. This is achieved by combining individual Mc@NLO simulations [40] with suitably modified truncated parton showers. The actual implementation takes advantage of the various matrix element and parton shower generators which are implemented in the event generation framework SHERPA [41, 42]. The main aim of our work is to reduce renormalisation and factorisation scale dependence of the fixed-order part in the simulation.

We have elucidated the new merging technique, MEPs@NLO, for electron-positron annihilation in a parallel publication [43]. The present paper is therefore organised as follows: section 2 reviews the basic formulae and discusses their extension to hadron-hadron collisions. For a more pedagogical introduction of the new method, based on the case of $e^{-} e^{+}$annihilations into hadrons, the reader is referred to the parallel publication, [43]. Section 3 details the Monte-Carlo implementation of our method. Section 4 presents first applications of the procedure to $W$-production at the Large Hadron Collider. Section 5 contains our conclusions and an outlook.

\section{Multijet merging at next-to leading order - MEPs@NLO}

As the scope of this publication will be to extend the method presented in [43] to hadron collisions, let us briefly discuss the difference between initial- and final-state parton evolution. Combining the two in a common formalism allowed an easy proof of logarithmic accuracy for the leading-order merging methods described in [35, 44]. The exact same arguments will hold in the next-to-leading order case.

Parton showers have two main ingredients, the differential branching probability $\mathcal{P}_{a b}$ for a parton of flavour $a$ to branch and produce a new parton $b$, and the momentum mapping, also called the recoil scheme, which describes how a final state of $n$ on-shell partons is transformed into a final state with $(n+1)$ on-shell partons after the emission. The differential branching probability lends itself into a no-branching probability, $\Delta$, also called the Sudakov factor of the parton shower.

In the case of leptonic initial states, parton evolution takes place solely in the final state, and it is therefore unconstrained. The corresponding Sudakov factors represent unconstrained no-branching probabilities

$$
\Delta\left(t, t^{\prime}\right)=\exp \left\{-\int_{t^{\prime}}^{t} \mathrm{~d} \bar{t} \int_{z_{\min }}^{z_{\max }} \mathrm{d} \bar{z} \frac{\alpha_{s}(\bar{z}, \bar{t})}{2 \pi \bar{t}} \mathcal{P}_{a b}(\bar{z}, \bar{t})\right\}
$$


They fulfill the simple relation $\Delta\left(t, t^{\prime}\right)=\Delta\left(t_{c}, t\right) / \Delta\left(t_{c}, t^{\prime}\right)$, where $t_{c}$ is an auxiliary scale, commonly taken to be the infrared cutoff scale of the evolution.

In the case of hadronic initial states, the incoming partons undergo a constrained evolution, with the boundary condition being energy-momentum sum rules for the proton. There are two possible formulations of the respective no-branching probability, which can be interchanged and which are fully equivalent $[45,46]$.

$$
\Delta\left(x, t, t^{\prime}\right)=\frac{f_{a}(x, t)}{f_{a}\left(x, t^{\prime}\right)} \frac{\Delta\left(t_{c}, t^{\prime}\right)}{\Delta\left(t_{c}, t\right)}=\exp \left\{-\int_{t^{\prime}}^{t} \mathrm{~d} \bar{t} \int_{x}^{z_{\max }} \mathrm{d} \bar{z} \frac{\alpha_{s}(\bar{z}, \bar{t})}{2 \pi \bar{t}} \mathcal{P}_{a b}(\bar{z}, \bar{t}) \frac{f_{b}(x / \bar{z}, \bar{t})}{\bar{z} f_{a}(x, \bar{t})}\right\} .
$$

Equation (2.2) is easy to interpret. When an initial-state parton undergoes branching, sum rules must be respected, and therefore the branching probability is reduced or enhanced by a factor proportional to the parton densities in the initial-state hadron before and after the splitting. In the following, we will only work with the second formulation, as it allows to write the evolution kernel in the same manner for initial- and final-state evolution. That is, we define the combined evolution kernel for an $n$-parton state with $m$ initial-state partons as follows:

$$
\mathrm{K}_{n}\left(\Phi_{1}\right)=\sum_{i=1}^{m} \frac{\alpha_{s}\left(z_{i}, t_{i}\right)}{2 \pi t_{i}} \mathcal{P}_{b_{i} a_{i}}\left(z_{i}, t_{i}\right) \frac{f_{b}\left(x_{i} / z_{i}, t_{i}\right)}{z_{i} f_{a}\left(x_{i}, t_{i}\right)}+\sum_{i=m+1}^{n+2} \frac{\alpha_{s}\left(z_{i}, t_{i}\right)}{2 \pi t_{i}} \mathcal{P}_{a_{i} b_{i}}\left(z_{i}, t_{i}\right) .
$$

Note that the definition of $z_{i}$ and $t_{i}$ as functions of the phase space variables of the emission, $\Phi_{1}$, depends on the choice of evolution or ordering parameter and the specifics of the recoil scheme of the shower.

\subsection{Ingredients of the merging method}

In order to describe the merging of next-to-leading order matrix elements, starting from a core $n$-parton process, the following quantities need to be introduced:

a) Hardness measure $Q$ and phase space regimes. A measure $Q_{i j}$, defined on any pair of partons $i$ and $j$ in both final- and initial-state is used to define their relative phase space distance. Thus, any $(n+k)$-parton final state can be assigned a unique value $Q_{n+k}=Q\left(\Phi_{n+k}\right)$, equal to the minimum of all $Q_{i j}$ constructed from the $n+k$ partons, including initial states. This value can be below or above a predefined value $Q_{\text {cut }}$, called the merging scale. The merging scale acts as an infrared cutoff on all $n+k$-jet calculations.

b) Squared leading-order (Born) matrix elements, $\mathrm{B}_{n+k}\left(\Phi_{n+k}\right)$, for $n+k$ outgoing particles, summed (averaged) over final state (initial state) spins and colours, including symmetry and flux factors and multiplied by parton luminosities. The corresponding real-emission matrix elements are called $\mathrm{R}_{n+k}\left(\Phi_{n+k+1}\right)$. Note that $\mathrm{R}_{n+k}\left(\Phi_{n+k+1}\right)=\mathrm{B}_{n+k+1}\left(\Phi_{n+k+1}\right)$. For details see [39].

c) Sudakov factors of the parton shower for the evolution kernel $\mathrm{K}_{n+k}$, which are given by

$$
\Delta_{n+k}^{(\mathrm{PS})}\left(t, t^{\prime}\right)=\exp \left\{-\int_{t}^{t^{\prime}} \mathrm{d} \Phi_{1} \mathrm{~K}_{n+k}\left(\Phi_{1}\right)\right\} \text {. }
$$


They include parton luminosities for initial state legs, as detailed in eq. (2.3). The one-emission phase-space element for the splitting, $\mathrm{d} \Phi_{1}$, is parametrised as

$$
\mathrm{d} \Phi_{1} \propto \mathrm{d} t \mathrm{~d} z \mathrm{~d} \phi,
$$

where $t$ is the ordering variable, $z$ is the splitting variable and $\phi$ is the azimuthal angle.

d) The resummation scale $\mu_{Q}$, which defines an upper limit of parton evolution for the core process with multiplicity $n$.

e) The MC@NLO method of [40] is used to match each $n+k$-parton NLO matrix element to parton showers individually. This implies MC@NLO evolution kernels $\mathrm{D}_{n+k}^{(\mathrm{A})} / \mathrm{B}_{n+k}^{(\mathrm{A})}$ as introduced in [40]. They account for the first (hardest) emission in the MC@NLO generator, which is produced with a modified Sudakov factor, $\Delta_{n+k}^{(\mathrm{A})}\left(t, t_{n+k}\right)$. In our implementation, the kernels carry full colour information, which is needed to maintain NLO accuracy [40].

f) The NLO-weighted Born differential cross sections $\overline{\mathrm{B}}_{n+k}^{(\mathrm{A})}$ and $\tilde{\mathrm{B}}_{n+k}^{(\mathrm{A})}$,

$$
\begin{aligned}
& \overline{\mathrm{B}}_{n+k}^{(\mathrm{A})}\left(\Phi_{n+k}\right)=\mathrm{B}_{n+k}\left(\Phi_{n+k}\right)+\tilde{\mathrm{V}}_{n+k}\left(\Phi_{n+k}\right)+\mathrm{I}_{n+k}^{(\mathrm{S})}\left(\Phi_{n+k}\right) \\
& +\int \mathrm{d} \Phi_{1}\left[\mathrm{D}_{n+k}^{(\mathrm{A})}\left(\Phi_{n+k+1}\right) \Theta\left(t_{n+k}-t_{n+k+1}\right)-\mathrm{D}_{n+k}^{(\mathrm{S})}\left(\Phi_{n+k+1}\right)\right] \\
& \tilde{\mathrm{B}}_{n+k}^{(\mathrm{A})}\left(\Phi_{n+k}\right)=\overline{\mathrm{B}}_{n+k}^{(\mathrm{A})}\left(\Phi_{n+k}\right) \\
& \quad+\mathrm{B}_{n+k}\left(\Phi_{n+k}\right) \sum_{i=n}^{n+k-1} \int \mathrm{d} \Phi_{1} \mathrm{~K}_{i}\left(\Phi_{1}\right) \Theta\left(t_{i}-t_{n+k+1}\right) \Theta\left(t_{n+k+1}-t_{i+1}\right) .
\end{aligned}
$$

While the $\overline{\mathrm{B}}_{n+k}^{(\mathrm{A})}$ are the regular NLO-weighted differential cross sections of the MC@NLO method [40], $\tilde{\mathrm{B}}_{n+k}^{(\mathrm{A})}$ also take into account emissions introduced by the parton shower above the last hard scale identified in the matrix element, $t_{n}$. They populate different regions of phase space, as indicated by the $\Theta$-functions in the evolution parameter $t_{n+k+1}$ of the respective emission. Compared to a standard MC@NLO simulation their inclusion is necessary because truncated parton shower emissions alter the real-radiation pattern at $\mathcal{O}\left(\alpha_{s}\right)$. Further, $\tilde{\mathrm{V}}_{n+k}$ is the Born-contracted collinearsubtracted one-loop amplitude, while the sum of integrated subtraction terms is given by $\mathrm{I}_{n+k}^{(\mathrm{S})}$. The latter correspond to the real subtraction terms $\mathrm{D}_{n+k}^{(\mathrm{S})}$, which can be decomposed in terms of individual dipole contributions, $\mathrm{D}=\sum_{i j, k} \mathrm{D}_{i j, k}[47,48]$. The $\mathrm{D}_{n+k}^{(\mathrm{A})}$ again are the MC@NLO evolution kernels. 
g) The hard remainder functions

$$
\begin{aligned}
\mathrm{H}_{n+k}^{(\mathrm{A})}\left(\Phi_{n+k+1}\right)= & \mathrm{R}_{n+k}\left(\Phi_{n+k+1}\right)-\mathrm{D}_{n+k}^{(\mathrm{A})}\left(\Phi_{n+k+1}\right) \Theta\left(t_{n+k}-t_{n+k+1}\right) \\
\tilde{\mathrm{H}}_{n+k}^{(\mathrm{A})}\left(\Phi_{n+k+1}\right)= & \mathrm{H}_{n+k}^{(\mathrm{A})}\left(\Phi_{n+k+1}\right) \\
& -\mathrm{B}_{n+k}\left(\Phi_{n+k}\right) \sum_{i=n}^{n+k-1} \mathrm{~K}_{i}\left(\Phi_{1}\right) \Theta\left(t_{i}-t_{n+k+1}\right) \Theta\left(t_{n+k+1}-t_{i+1}\right),
\end{aligned}
$$

which contain both the standard MC subtraction terms, $\mathrm{D}_{n+k}^{(\mathrm{A})}$, and the additional subtraction terms for the parton shower above $t_{n}$.

h) The compound subtraction term

$$
\tilde{\mathrm{D}}_{n+k}^{(\mathrm{A})}\left(\bar{\Phi}_{1}\right)=\mathrm{D}_{n+k}^{(\mathrm{A})} \Theta\left(t_{n+k}-\bar{t}\right)+\mathrm{B}_{n+k} \sum_{i=n}^{n+k-1} \mathrm{~K}_{i} \Theta\left(t_{i}-\bar{t}\right) \Theta\left(\bar{t}-t_{i+1}\right),
$$

which is used to simplify the above expressions for $\tilde{\mathrm{B}}_{n+k}^{(\mathrm{A})}$ and $\tilde{\mathrm{H}}_{n+k}^{(\mathrm{A})}$. It leads to a compound evolution kernel defining the compound Sudakov factor

$$
\begin{aligned}
\tilde{\Delta}_{n+k}^{(\mathrm{A})}\left(t, t^{\prime}\right) & =\exp \left\{-\int_{t}^{t^{\prime}} \mathrm{d} \bar{\Phi} \frac{\tilde{\mathrm{D}}_{n+k}^{(\mathrm{A})}}{\mathrm{B}_{n+k}}\right\} \\
& =\exp \left\{-\int_{t}^{t^{\prime}} \mathrm{d} \bar{\Phi} \frac{\mathrm{D}_{n+k}^{(\mathrm{A})}}{\mathrm{B}_{n+k}} \Theta\left(t_{n+k}-\bar{t}\right)\right\} \times \\
& \times \prod_{i=n}^{n+k-1} \exp \left\{-\int_{t}^{t^{\prime}} \mathrm{d} \bar{\Phi} \mathrm{K}_{i} \Theta\left(t_{i}-\bar{t}\right) \Theta\left(\bar{t}-t_{i+1}\right)\right\} \\
& =\Delta_{n+k}^{(\mathrm{A})}\left(t, t^{\prime}\right) \prod_{i=n}^{n+k-1} \Delta_{i}^{(\mathrm{PS})}\left(t, t^{\prime}\right) .
\end{aligned}
$$

Correspondingly, a compound Sudakov factor for the parton shower is given by

$$
\tilde{\Delta}_{n+k}^{(\mathrm{PS})}\left(t, t^{\prime}\right)=\prod_{i=n}^{n+k} \exp \left\{-\int_{t}^{t^{\prime}} \mathrm{d} \bar{\Phi}_{1} \mathrm{~K}_{i} \Theta\left(t_{i}-\bar{t}\right) \Theta\left(\bar{t}-t_{i+1}\right)\right\}=\prod_{i=n}^{n+k} \Delta_{i}^{(\mathrm{PS})}\left(t, t^{\prime}\right) .
$$

The compound Sudakov factors describe the evolution from $t^{\prime}$ to $t$, embedding it into the emission structure which is predefined by the hard matrix elements in the respective $n+k$ configuration. For details on how to construct this structure, see [35].

One could identify the hardness measure $Q$ of item a) with the parton shower evolution variable $t$. If this is not done, for example in the case of an angular ordered parton shower 
and a hardness measure corresponding to transverse momenta, a truncated parton shower must be used to ensure that the emission phase space is properly filled [23, 35]. This is the case for example in HERWIG, where the evolution is formulated in terms of generalised relative angles $[49,50]$.

Sudakov form factors are split into two regimes, a hard one $\left(>Q_{\text {cut }}\right)$ and a soft one $\left(<Q_{\text {cut }}\right)$ such that

$$
\Delta^{(\mathrm{PS})}\left(t, t^{\prime} ;<Q_{\text {cut }}\right) \Delta^{(\mathrm{PS})}\left(t, t^{\prime} ;>Q_{\text {cut }}\right)=\Delta^{(\mathrm{PS})}\left(t, t^{\prime}\right)
$$

Pictorially speaking these two regions can be identified with emissions that populate the matrix element and the parton shower region, respectively. In the case where evolution variable and hardness measure coincide, it is not possible to produce emissions $<Q_{\text {cut }}$ in the matrix element region, therefore $\Delta^{(\mathrm{PS})}\left(t, t^{\prime} ;<Q_{\text {cut }}\right)=\Delta^{(\mathrm{PS})}\left(t, t^{\prime} ;<t_{\text {cut }}\right)=1$. In this case the Sudakov factors $\prod_{i=n}^{n+k-1} \Delta_{i}^{(\mathrm{PS})}\left(t, t^{\prime}\right)$ of eqs. (2.9) and (2.10) should be interpreted as pure vetos in the kinematic regime of the intermediate states. If, on the other hand, there is a mismatch between evolution variable and hardness parameter, there are two possibilities to implement emissions with $Q<Q_{\text {cut }}$ that have an ordering parameter larger than the smallest one identified in the matrix element. Either one chooses to veto matrix element configurations where the boundary between matrix element and parton shower regions would be crossed multiple times and fills these regions entirely with the parton shower [32,37]. Or one implements the additional intermediate branchings, thereby amending momentum and colour topology of the final state [35, 36]. The formalism for merging NLO matrix elements presented here works for both of these methods.

\subsection{Definition of the merging method}

We define our algorithm in terms of the expectation value of an arbitrary, infrared-safe observable $O$, evaluated by taking the average over sufficiently many points in the multiparton phase-space. This will help us to show, by explicit calculations, that our method indeed satisfies the two formal requirements for a correct multijet merging:

1. The formal perturbative accuracy of the matrix elements is preserved - in our case this translates into ensuring that the jet cross section for any given $n+k$-jet final state is described at NLO accuracy and that the first emission is LO correct. This means that all terms which are parametrically $\mathcal{O}\left(\alpha_{s}\right)$ with respect to the Born configuration are unaltered.

2. The formal accuracy of the parton shower is guaranteed. This means that the logarithmic structure of the evolution is ensured, or, in other words, the matrix elements do not introduce new logarithms at an accuracy level maintained by the parton shower.

To be more concrete, let us assume in the following that $m$ next-to-leading order predictions are merged, starting with an $n$-particle final state, and that $p$ leading-order predictions are added on top. We consider an observable which is sensitive to at least 
$(n+k)$ partons. This observable is determined as

$$
\langle O\rangle=\sum_{j=0}^{\infty}\left\langle O_{j}\right\rangle=\sum_{i=0}^{\infty}\left\langle O_{n+k+i}\right\rangle, \quad \text { i.e. } \quad\left\langle O_{j}\right\rangle \equiv 0 \quad \forall j<n+k
$$

where $\left\langle O_{j}\right\rangle$ are the contributions from $j$-parton final states. The respective partons can be generated through either the parton-level matrix elements or the shower algorithm. We will not explicitly mention any parton-shower emissions beyond those also described by the fixed-order calculations whose accuracy we intend to maintain. For simplicity we assume that the $(n+k)$-particle final state is always described at next-to-leading order in the strong coupling, i.e. $k \leq m$. Thus, for the observable $O$ above, we have to trace up to three terms in the case that $(n+k+1)$-particle final states are generated at next-to-leading order $(k<m)$ and up to two terms in the case that they are generated at leading order $(k=m)$. The latter case was analysed extensively in [39] and shall not be reviewed here. In the following we will focus solely on the scenario that the $(n+k+1)$-particle final state (and the corresponding $(n+k+2)$-particle real-emission contribution) is simulated with an NLO parton-level generator.

It is important to distinguish between partons and parton-level jets. We do not consider jets as defined experimentally, using any existing jet algorithm, but introduce a separate parton-level definition which is needed simply to separate parts of our inclusive event samples. To this end, we use the hardness measure $Q_{n+k}$ of item a) section 2.1 as a jet criterion. This value can be below or above the merging scale $Q_{\text {cut }}$, where for $Q_{n+k}>Q_{\text {cut }}$, $\Phi_{n+k}$ is called an $(n+k)$-jet configuration.

In the context of next-to-leading order calculations, one also needs to define a criterion for the corresponding real-emission configuration to be of $(n+k)$-jet type. To this end, we reduce the $(n+k+1)$-parton final state to an $(n+k)$-parton final state by backward clustering according to the kinematics of the $\tilde{\mathrm{D}}_{n+k}^{(\mathrm{A})}$. The pair of partons to be clustered and the corresponding spectator parton are identified to be the ones leading to the smallest jet measure.

To simplify our notation, we define the $j$-jet inclusive and exclusive expectation values

$$
\begin{aligned}
\langle O\rangle_{j}^{\text {incl }} & =\sum_{l=0}^{\infty}\left\langle O_{j+l} \Theta\left(Q_{j}-Q_{\text {cut }}\right)\right\rangle, \\
\langle O\rangle_{j}^{\text {excl }} & =\sum_{l=0}^{\infty}\left\langle O_{j+l} \Theta\left(Q_{j}-Q_{\text {cut }}\right) \Theta\left(Q_{\text {cut }}-Q_{j+1}\right)\right\rangle .
\end{aligned}
$$

They include contributions from all final states with at least $j$ partons, which must form $j$ (but not $j+1$ in the case of $\langle O\rangle_{j}^{\text {excl }}$ ) parton-level jets according to the definition of $Q$. The $j+l$-parton configuration is reduced to a $j$-parton configuration for the purpose of jet identification by an exact inverse parton shower [35].

With these definitions at hand, the MEPs@NLO method can be formulated in terms of $(n+k)$-jet exclusive expectation values of generic infrared safe observables. To $\mathcal{O}\left(\alpha_{s}\right)$ it 
reads

$$
\begin{aligned}
& \langle O\rangle_{n+k}^{\text {excl }}=\int \mathrm{d} \Phi_{n+k} \Theta\left(Q_{n+k}-Q_{\text {cut }}\right) \tilde{\mathrm{B}}_{n+k}^{(\mathrm{A})} \\
& \quad \times\left[\tilde{\Delta}_{n+k}^{(\mathrm{A})}\left(t_{c}, \mu_{Q}^{2}\right) O_{n+k}\right. \\
& \quad+\int_{t_{c}}^{t_{n+k}} \mathrm{~d} \Phi_{1} \frac{\left.\tilde{\mathrm{D}}_{n+k}^{(\mathrm{A})} \tilde{\mathrm{B}}_{n+k}^{(\mathrm{A})}\left(t_{n+k+1}, \mu_{Q}^{2}\right) \Theta\left(Q_{\text {cut }}-Q_{n+k+1}\right) O_{n+k+1}\right]}{+\int \mathrm{d} \Phi_{n+k+1} \Theta\left(Q_{n+k}-Q_{\text {cut }}\right) \tilde{\mathrm{H}}_{n+k}^{(\mathrm{A})} \tilde{\Delta}_{n+k}^{(\mathrm{PS})}\left(t_{n+k+1}, \mu_{Q}^{2} ;>Q_{\text {cut }}\right) \times} \\
& \quad \times \Theta\left(Q_{\text {cut }}-Q_{n+k+1}\right) O_{n+k+1},
\end{aligned}
$$

where $t_{c}$ is the infrared cutoff of the parton shower and $t_{n+k}$ is the starting scale on the $n+k$ parton ensemble. Hence $t_{n}=\mu_{Q}^{2}$ and $t_{n+k}(k>1)$ is the smallest phase space distance in the parton shower evolution variable $t$, i.e. the emission scale of the $(n+k)$ th parton on the $n+k-1$ parton ensemble. $t_{n+k+1}$ then is the emission scale of the $(n+k+1)$ th parton. Phase space configurations breaking the scale hierarchy $t_{n}>t_{n+1}>\ldots t_{n+k-1}>t_{n+k}$ are described by the so-called hard events implemented in the second term. In this case no resummation of emission scales is effected.

Note that there is a slight mismatch between the terms $\tilde{\mathrm{D}}_{n+k}^{(\mathrm{A})}$ in the NLO-weighted Born-level cross section and hard remainder on one hand and the actual emission terms $\tilde{\mathrm{B}}_{n+k}^{(\mathrm{A})} \cdot \tilde{\mathrm{D}}_{n+k}^{(\mathrm{A})} / \mathrm{B}_{n+k}$ on the other hand. It generates corrections of higher order in $\alpha_{s}$, and thus can safely be ignored. This is the same reasoning as in the MC@NLO method [17].

Eq. (2.14) can be written in a more suitable form by factorising the compound Sudakov factors in analogy to eq. (2.11)

$$
\begin{aligned}
\langle O\rangle_{n+k}^{\text {excl }}= & \int \mathrm{d} \Phi_{n+k} \Theta\left(Q_{n+k}-Q_{\text {cut }}\right) \tilde{\mathrm{B}}_{n+k}^{(\mathrm{A})} \tilde{\Delta}_{n+k}^{(\mathrm{A})}\left(t_{c}, \mu_{Q}^{2} ;>Q_{\text {cut }}\right) \\
\times & {\left[\tilde{\Delta}_{n+k}^{(\mathrm{A})}\left(t_{c}, \mu_{Q}^{2} ;<Q_{\text {cut }}\right) O_{n+k}\right.} \\
& \left.+\int_{t_{c}}^{t_{n+k}} \mathrm{~d} \Phi_{1} \frac{\tilde{\mathrm{D}}_{n+k}^{(\mathrm{A})}}{\mathrm{B}_{n+k}} \tilde{\Delta}_{n+k}^{(\mathrm{A})}\left(t_{n+k+1}, \mu_{Q}^{2} ;<Q_{\text {cut }}\right) \Theta\left(Q_{\text {cut }}-Q_{n+k+1}\right) O_{n+k+1}\right] \\
+ & \int \mathrm{d} \Phi_{n+k+1} \Theta\left(Q_{n+k}-Q_{\text {cut }}\right) \tilde{\mathrm{H}}_{n+k}^{(\mathrm{A})} \tilde{\Delta}_{n+k}^{(\mathrm{PS})}\left(t_{n+k+1}, \mu_{Q}^{2} ;>Q_{\text {cut }}\right) \times \\
& \quad \times \Theta\left(Q_{\text {cut }}-Q_{n+k+1}\right) O_{n+k+1} .
\end{aligned}
$$

It is now explicit that the Sudakov form factor on the first line accounts for a veto on emissions with $Q>Q_{\text {cut }}$. This is completely equivalent to the MEPs method [35]. If the definition of hardness, $Q$, and the evolution parameter of the parton shower, $t$, coincide, 
we obtain in the same manner

$$
\begin{aligned}
\langle O\rangle_{n+k}^{\text {excl }}=\int & \mathrm{d} \Phi_{n+k} \Theta\left(t_{n+k}-t_{\text {cut }}\right) \tilde{\mathrm{B}}_{n+k}^{(\mathrm{A})}\left(\prod_{i=n}^{n+k-1} \Delta_{i}^{(\mathrm{PS})}\left(t_{i+1}, t_{i}\right)\right) \\
& \times\left[\Delta_{n+k}^{(\mathrm{A})}\left(t_{c}, t_{n+k}\right) O_{n+k}\right. \\
& \left.\quad+\int_{t_{c}}^{t_{n+k}} \mathrm{~d} \Phi_{1} \frac{\mathrm{D}_{n+k}^{(\mathrm{A})}}{\mathrm{B}_{n+k}} \Delta_{n+k}^{(\mathrm{A})}\left(t_{n+k+1}, t_{n+k}\right) \Theta\left(t_{\text {cut }}-t_{n+k+1}\right) O_{n+k+1}\right] \\
+ & \int \mathrm{d} \Phi_{n+k+1} \Theta\left(t_{n+k}-t_{\text {cut }}\right) \mathrm{H}_{n+k}^{(\mathrm{A})}\left(\prod_{i=n}^{n+k} \Delta_{i}^{(\mathrm{PS})}\left(t_{i+1}, t_{i}\right)\right) \times \\
& \times \Theta\left(t_{\text {cut }}-t_{n+k+1}\right) O_{n+k+1},
\end{aligned}
$$

where $t_{\text {cut }}=Q_{\text {cut }}$, reproducing the expressions of [43]. At the same time, $\tilde{\mathrm{H}}_{n+k}^{(\mathrm{A})}$ has been replaced by $\mathrm{H}_{n+k}^{(\mathrm{A})}$, since the two coincide if $t_{n+k}>t_{\text {cut }}$ and $t_{n+k+1}<t_{\text {cut }}$, which is enforced by the two $\Theta$-functions on the last line. Further, combining the additional terms of the $\tilde{\mathrm{B}}_{n+k}^{(\mathrm{A})}$ function, cf. eq. (2.6), with the Sudakov factor implementing vetoed emissions on the first line renders the form of the $\mathcal{O}\left(\alpha_{s}\right)$ correction more explicit

$$
\begin{aligned}
\langle O\rangle_{n+k}^{\mathrm{excl}}=\int \mathrm{d} \Phi_{n+k} \Theta\left(t_{n+k}-t_{\text {cut }}\right) \overline{\mathrm{B}}_{n+k}^{(\mathrm{A})}\left[\prod_{i=n}^{n+k-1} \Delta_{i}^{(\mathrm{PS})}\left(t_{i+1}, t_{i}\right)\left(1+\frac{\mathrm{B}_{n+k}}{\overline{\mathrm{B}}_{n+k}^{(\mathrm{A})}} \int_{t_{i+1}}^{t_{i}} \mathrm{~d} \Phi_{1} \mathrm{~K}_{i}\right)\right] \\
\times\left[\Delta_{n+k}^{(\mathrm{A})}\left(t_{c}, t_{n+k}\right) O_{n+k}\right. \\
\left.\quad+\int_{t_{c}}^{t_{n+k}} \mathrm{~d} \Phi_{1} \frac{\mathrm{D}_{n+k}^{(\mathrm{A})}}{\mathrm{B}_{n+k}} \Delta_{n+k}^{(\mathrm{A})}\left(t_{n+k+1}, t_{n+k}\right) \Theta\left(t_{\mathrm{cut}}-t_{n+k+1}\right) O_{n+k+1}\right] \\
+\int \mathrm{d} \Phi_{n+k+1} \Theta\left(t_{n+k}-t_{\text {cut }}\right) \mathrm{H}_{n+k}^{(\mathrm{A})}\left(\prod_{i=n}^{n+k} \Delta_{i}^{(\mathrm{PS})}\left(t_{i+1}, t_{i}\right)\right) \times \\
\quad \times \Theta\left(t_{\text {cut }}-t_{n+k+1}\right) O_{n+k+1} .
\end{aligned}
$$

Note that we have added arbitrary higher-order terms, which allow to include the sum over truncated shower subtractions in $\overline{\mathrm{B}}_{n+k}^{(\mathrm{A})}$ in the product in line one.

The various terms are interpreted easily. The products in the square brackets on the first line constitute truncated vetoed parton showers, with their $\mathcal{O}\left(\alpha_{s}\right)$ terms partially subtracted. In practice, these expressions can be generated by running a truncated vetoed shower and skipping/reweighting the first veto, depending on $\mathrm{B}_{n+k} / \overline{\mathrm{B}}_{n+k}^{(\mathrm{A})}$. The remainder of the expression corresponds to an ordinary MC@NLO simulation, consisting of standard $(\mathbb{S})$ and hard $(\mathbb{H})$ events. This scheme is particularly easy to implement in practice, because no emissions need to be generated in the truncated shower. This version of the method has been explained in detail in [43]. 


\subsection{Fixed-order and logarithmic accuracy}

The proof of next-to-leading order and logarithmic accuracy of the above described MEPS@NLO method in $e^{+} e^{-}$-collisions was presented in [43], for the simpler case where truncated shower emissions are absent. The proof presented here extends to the case where they are included.

Proving fixed order accuracy is a fairly straightforward exercise. For this it is sufficient to expand eq. (2.14) to the first order in $\alpha_{s}$. By construction, this yields exactly the same result as is obtained in MC@NLO for $Q_{n+k+1}<Q_{\text {cut }}$, i.e. $\langle O\rangle_{n+k}^{\text {excl }}$. The effects of Sudakov suppression and modified subtraction cancel to first order in $\alpha_{s}$, and the correct fixed-order radiation pattern is recovered.

The proof of logarithmic accuracy inherent to the parton shower is a bit more cumbersome as inclusive observables have to be considered. It proceeds as follows:

1) Below $Q_{\text {cut }}$ the method proposed here reproduces the formal accuracy of a corresponding MC@NLO approach for $(n+k)$ jets. MC@NLO guarantees that the first emission follows the corresponding tree-level matrix element for $(n+k+1)$ final state particles and that $t_{n+k+1}$ is resummed according to the modified Sudakov factor $\Delta_{n+k}^{(\mathrm{A})}\left(t_{n+k+1}, t_{n+k}\right)$. Further emissions of course are generated by the parton shower and therefore, by construction, exhibit the correct behaviour.

2) One needs to show that above $Q_{\text {cut }}$ the combination of the $(n+k)$-jet and $(n+k+1)$ jet exclusive samples does not generate unwanted terms in the $(n+k+1)$-parton ensemble. As explained in 1), in the $(n+k)$-jet contribution the second emission is generated by the parton shower and therefore correct by construction. It remains to show that the $(n+k+1)$-jet contribution does not generate any logarithmic corrections beyond the formal accuracy of the parton shower. This is guaranteed since the $(n+k+2)$ th parton is generated through MC@NLO techniques, which, again by construction, maintain the logarithmic accuracy of the parton shower.

3) Within the $(n+k+1)$-jet exclusive sample the same considerations as for 1) apply. Thus, the correct resummation of scale hierarchies is achieved. The subsequent merging of the $(n+k+2)$-jet exclusive sample proceeds as in 2).

To formally prove 1) and 2) we decompose the contribution to the generic $(n+k)$-jet inclusive observable as follows

$$
\langle O\rangle_{n+k}^{\mathrm{incl}}=\langle O\rangle_{n+k}^{\mathrm{MCNLO}}+\langle O\rangle_{n+k}^{\mathrm{corr}} .
$$

The MC@NLO result $\langle O\rangle_{n+k}^{\mathrm{MCNLO}}$ is obtained by dropping the constraint $\Theta\left(Q_{\text {cut }}-Q_{n+k+1}\right)$ 
in eq. (2.14).

$$
\begin{aligned}
\langle O\rangle_{n+k}^{\mathrm{MC} @ \mathrm{NLO}}=\int \mathrm{d} \Phi_{n+k} \Theta\left(Q_{n+k}-Q_{\mathrm{cut}}\right) \tilde{\mathrm{B}}_{n+k}^{(\mathrm{A})} & \\
& \times\left[\tilde{\Delta}_{n+k}^{(\mathrm{A})}\left(t_{c}, \mu_{Q}^{2}\right) O_{n+k}+\int_{t_{c}}^{\mu_{Q}^{2}} \mathrm{~d} \Phi_{1} \frac{\tilde{\mathrm{D}}_{n+k}^{(\mathrm{A})}}{\mathrm{B}_{n+k}} \tilde{\Delta}_{n+k}^{(\mathrm{A})}\left(t, \mu_{Q}^{2}\right) O_{n+k+1}\right] \\
& +\int \mathrm{d} \Phi_{n+k+1} \Theta\left(Q_{n+k}-Q_{\text {cut }}\right) \tilde{\mathrm{H}}_{n+k}^{(\mathrm{A})} \tilde{\Delta}_{n+k}^{(\mathrm{PS})}\left(t_{n+k+1}, \mu_{Q}^{2} ;>Q_{\text {cut }}\right) O_{n+k+1} .
\end{aligned}
$$

Thus, $\langle O\rangle^{\mathrm{MC} @ N L O}$ is indeed the MC@NLO result for the $n+k$-parton final state, up to a product of Sudakov form factors, cf. section 2.1 items $\mathrm{f}$ )-h), which describe the implementation of truncated vetoed parton showers on the underlying Born configurations, starting from the $n$-particle final state.

From eq. (2.14), the correction term $\langle O\rangle_{n+k}^{\text {corr }}$ for the $(n+k+1)$-parton vanishes below $Q_{\text {cut }}$, completing part 1) of the proof. Above the merging cut, up to contributions of order $\alpha_{s}^{2}$, and ignoring the effect of emissions below $t_{n+k+1}$, it reads

$$
\begin{aligned}
\langle O\rangle_{n+k}^{\mathrm{corr}}=\int \mathrm{d} \Phi_{n+k+1} & \Theta\left(Q_{n+k+1}-Q_{\text {cut }}\right) \tilde{\Delta}_{n+k+1}^{(\mathrm{PS})}\left(t_{c}, \mu_{Q}^{2}\right) O_{n+k+1} \\
\times & \left\{\tilde{\mathrm{D}}_{n+k}^{(\mathrm{A})}\left[1-\frac{\tilde{\mathrm{B}}_{n+k}^{(\mathrm{A})}}{\mathrm{B}_{n+k}} \frac{\Delta_{n+k}^{(\mathrm{A})}\left(t_{n+k+1}, t_{n+k}\right)}{\Delta_{n+k}^{(\mathrm{PS})}\left(t_{n+k+1}, t_{n+k}\right)}\right]\right. \\
& \left.-\mathrm{B}_{n+k+1}\left[1-\frac{\tilde{\mathrm{B}}_{n+k+1}^{(\mathrm{A})}}{\mathrm{B}_{n+k+1}} \frac{\Delta_{n+k+1}^{(\mathrm{A})}\left(t_{c}, t_{n+k+1}\right)}{\Delta_{n+k+1}^{(\mathrm{PS})}\left(t_{c}, t_{n+k+1}\right)}\right]\right\} .
\end{aligned}
$$

The relevant terms to consider are the ones in the curly bracket. They must not upset the resummation properties of the $\langle O\rangle^{\mathrm{MC} @ N L O}$. Both terms consist of one factor directly responsible for the emission of an extra particle, $\tilde{\mathrm{D}}_{n+k}^{(\mathrm{A})}$ and $\mathrm{B}_{n+k+1}$, respectively, which will eventually yield a contribution of $\mathcal{O}\left(\alpha_{s} L^{2}\right)$. Analysing the factors multiplying these emission terms, reveals that each of them is at most of $\mathcal{O}\left(\alpha_{s} L\right)$. However, these logarithms, if present, are associated with a factor of $\frac{1}{N_{C}}$, they arise from the difference between the evolution kernels in $\mathrm{D}^{(\mathrm{A})}$ and $\mathrm{D}^{(\mathrm{PS})}=\mathrm{B} \cdot \mathrm{K}$. Clearly, the combined virtual and real contributions to $\tilde{\mathrm{B}}_{n+k}^{(\mathrm{A})}$ do not exhibit any logarithms that could upset the accuracy of the parton shower, because the phase space integrals over the real terms in eq. (2.6) are unrestricted. Taken together, this shows that the correction term does not upset the formal logarithmic accuracy of the parton shower for part 2) of the proof.

\subsection{Renormalisation and factorisation scale uncertainties}

The key aim of the MEPs@NLO approach presented here is to reduce the dependence of the merged prediction on the renormalisation scale $\mu_{R}$ and the factorisation scale $\mu_{F}$, which are employed in the computation of hard matrix elements. These scales have not been made explicit so far. 
Note that only the dependence on renormalisation and factorisation scales are reduced compared to the MEPS method, while the dependence on the resummation scale, $\mu_{Q}$, remains identical. This is a direct consequence of the fact that the parton-shower evolution is unchanged in our prescription. The resummation scale dependence was analysed in detail in [40].

Following the MEPs strategy, the renormalisation scale should be determined by analogy of the leading-order matrix element with the respective parton shower branching history [35]. In next-to-leading order calculations, however, one needs a definition which is independent of the parton multiplicity. The same scale should be used in Born matrix elements and real-emission matrix elements if they have similar kinematics, and in particular when the additional parton of the real-emission correction becomes soft or collinear. This can be achieved if we define the renormalisation scale for a process of $\mathcal{O}\left(\alpha_{s}^{n}\right)$ as

$$
\alpha_{s}\left(\mu_{R}^{2}\right)^{n}=\prod_{i=1}^{n} \alpha_{s}\left(\mu_{i}^{2}\right) .
$$

Here, $\mu_{i}^{2}$ are the respective scales defined by analogy of the Born configuration ${ }^{1}$ with a parton-shower branching history.

The renormalisation scale uncertainty in the MEPs@NLO approach is then determined by varying $\mu_{R} \rightarrow \tilde{\mu}_{R}$, while simultaneously correcting for the one-loop effects induced by a redefinition in eq. (2.21). That is, the Born matrix element is multiplied by

$$
\alpha_{s}\left(\tilde{\mu}_{R}^{2}\right)^{n}\left(1-\frac{\alpha_{s}\left(\tilde{\mu}_{R}^{2}\right)}{2 \pi} \beta_{0} \sum_{i=1}^{n} \log \frac{\mu_{i}^{2}}{\tilde{\mu}_{R}^{2}}\right),
$$

to generate the one-loop counterterm, while higher-order contributions remain the same.

Similar reasoning holds for the collinear mass factorisation counterterms. Given $\mu_{F}$ as determined by the MEPS algorithm, a different factorisation scale $\tilde{\mu}_{F}$ can be chosen, which leads to the $\mathcal{O}\left(\alpha_{s}\right)$ counterterm

$$
\begin{aligned}
\mathrm{B}_{n}\left(\Phi_{n}\right) \frac{\alpha_{s}\left(\tilde{\mu}_{R}^{2}\right)}{2 \pi} \log \frac{\mu_{F}^{2}}{\tilde{\mu}_{F}^{2}}\left(\sum_{c=q, g}^{n} \int_{x_{a}}^{1} \frac{\mathrm{d} z}{z} P_{a c}(z) f_{c}\left(x_{a} / z, \tilde{\mu}_{F}^{2}\right)\right. \\
\left.+\sum_{d=q, g}^{n} \int_{x_{b}}^{1} \frac{\mathrm{d} z}{z} P_{b d}(z) f_{d}\left(x_{b} / z, \tilde{\mu}_{F}^{2}\right)\right)
\end{aligned}
$$

where $a$ and $b$ denote the parton flavours of the initial state. The sums run over all parton flavours and $P(z)$ denote the regularised Altarelli-Parisi splitting kernels. In the case of only one hadronic initial state, one of the sums would be absent.

\section{Monte-Carlo implementation}

In this section we describe the Monte Carlo implementation of the merging formula eq. (2.14) in SHERPA. The techniques needed to combine leading-order matrix elements with parton showers are given elsewhere [35, 37].

\footnotetext{
${ }^{1}$ In the case of the real-emission correction and the corresponding dipole subtraction terms we consider the underlying Born configuration instead
} 
The algorithm reads as follows:

- Draw an event according to the total cross section of the inclusive NLO-expression, effectively a sum over $\overline{\mathrm{B}}^{(\mathrm{A})}$ and $\mathrm{H}_{n+k}$.

- According to the absolute value of the relative contributions, select a standard $(\mathbb{S})$ or hard $(\mathbb{H})$ event. Select flavours and momenta accordingly.

- Reconstruct a parton shower history over tree-level like configurations.

- Start the parton shower, which will work out differently for $\mathbb{S}$ and $\mathbb{H}$ events.

For the latter, perform a truncated and vetoed shower in the spirit of the LO MEPS method, cf. [35, 37]. If the matrix element level configuration could not be reached through standard parton showering (like, e.g. in the case of $u \bar{u} \rightarrow W^{+} s \bar{c}$ ) skip this step.

For the former, perform an MC@NLO step, i.e. generate an extra emission $t_{n+k+1}$ through the $\mathrm{D}_{n+k}^{(\mathrm{A})}$ terms. Perform a truncated and NLO-vetoed shower between $t_{n}$ and $t_{n+k}$. In contrast to a regular vetoed shower, in this NLO-vetoed shower, the first trial emission per cluster step, which is above $Q_{\text {cut }}$ will not lead to vetoing the event, but will be ignored, depending on the weight $\mathrm{B}_{n+k} / \overline{\mathrm{B}}_{n+k}^{(\mathrm{A})}$. This corresponds to the finite correction, i.e. the sum over the splitting kernels in (2.7). In [43], this corresponds to the correction term in the second line of eq. (3.5). There, we also described in more detail some of the implementation details.

For both types of events continue with a vetoed parton shower, as in the MEPs case.

\section{Results}

In this section we present results generated with the previously described merging method. We employ the leading-order matrix element generators AMEGIC++ [51] and Comix [52] in conjunction with the automated dipole subtraction provided in SHERPA [53] and the implementation of the Binoth-Les Houches interface [54] to obtain parton-level events at next-to-leading order. Virtual matrix elements for $W+n$ jets are provided by the BlACKHAT library $[6,9,55,56]$. We employ a parton shower based on Catani-Seymour dipole factorisation [57] and the related MC@NLO generator [40] to generate events at the parton shower level. In contrast to the other MC@NLO implementations, no leading colour approximation is made. Our generator therefore recovers the full next-to-leading order accuracy of the fixed-order result throughout the phase space, to all orders in the colour expansion.

We compare our predictions to a recent measurement [58] of $W+$ jets events by the ATLAS collaboration. The analysis is used as implemented in the Rivet [59] framework and selects events containing a lepton within $|\eta|<2.5$ with $p_{\perp}>20 \mathrm{GeV}$ and $E_{T}^{\text {miss }}>25 \mathrm{GeV}$. From the lepton and neutrino a transverse mass variable is calculated and required to fulfil $m_{\mathrm{T}}^{\mathrm{W}}>40 \mathrm{GeV}$. Jets are clustered using the anti- $k_{t}$ algorithm [60] in the implementation of FastJet [61] with $R=0.4$. We use the CT10 PDF set [62]. 

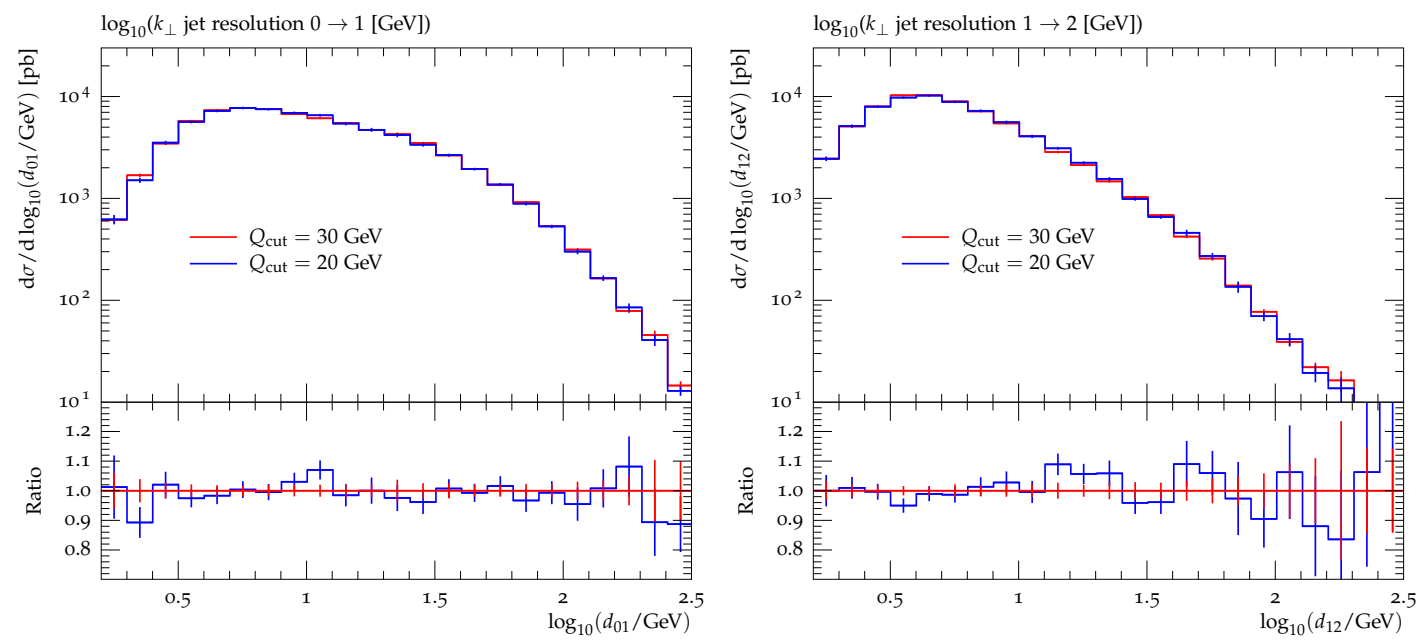

Figure 1. Stability of the predictions for the two hardest splitting scales in the $k_{T}$ cluster algorithm under variation of the merging cut $Q_{\text {cut }}$ from $30 \mathrm{GeV}$ to $20 \mathrm{GeV}$. The statistical errors are indicated.

Effects from hadronisation and multiple parton interactions are not taken into account in the scope of this publication but can easily be enabled on top of the parton shower level studies pursued here. This is also justified by the fact that the two effects happen to compensate each other to a large extent in this analysis.

SHERPA predictions are made in three different approaches:

MC@NLO NLO+PS matched sample for the $W+0$-jet process using the MC@NLO-like implementation described in [40].

MENLOPS The MENLOPS method described in [39] is used to merge an NLO+PS sample for the $W+0$-jet process with tree-level matrix elements for the higher multiplicity $W+1,2,3,4$-jet processes. Here we use this method on top of the $W+0$-jet MC@NLO sample, as described in more detail in [43].

MePs@NLO The MePs@NLO method was described in section 2 and is used here for the $W+0,1,2$-jet processes at NLO. In addition, the $W+3,4$-jet processes are merged using tree-level matrix elements via the MENLOPS technique.

For the two latter approaches the jet criterion $Q_{i j}$, separating matrix element and parton shower regions, is defined as

$$
Q_{i j}^{2}=2 p_{i} p_{j} \min _{k \neq i, j} \frac{2}{C_{i, j}^{k}+C_{j, i}^{k}} \quad \text { where } \quad C_{i, j}^{k}=\frac{p_{i} p_{k}}{\left(p_{i}+p_{k}\right) p_{j}},
$$

using $Q_{\text {cut }}=30 \mathrm{GeV}$ as default. In figure 1 the stability of the simulation under a variation of the merging cut $Q_{\text {cut }}$ is studied. Predictions are displayed for the two hardest splitting scales in a $k_{T}$ cluster sequence, which provides a very sensitive probe of merging-related artefacts. We compare here two MePs@NLO samples with $W+0,1$-jet production at NLO and the merging separation criterion set to $Q_{\text {cut }}=20,30 \mathrm{GeV}$ to assess the size of the 
unwanted terms of higher logarithmic order in eq. (2.20). The event samples are statistically compatible, with merging-related effects up to approximately $5 \%$ in the vicinity of $Q_{\text {cut }}$.

We now turn to study perturbative uncertainties stemming from variations of the factorisation and renormalisation scale in the matrix elements. The scales are chosen by clustering the $2 \rightarrow n$ parton level kinematics onto a core $2 \rightarrow 2$ configuration using a $k_{T^{-}}$ type algorithm with recombination into on-shell particles. The central scale $\mu_{F}=\mu_{R}=\mu$ is then defined as the lowest invariant mass or virtuality in the core process. For core interactions which are pure QCD processes scales are set to the maximum transverse mass squared of the outgoing particles. A variation by a factor of two in each direction as $\mu_{F}=\mu_{R}=\frac{\mu}{2} \ldots 2 \cdot \mu$ generates the uncertainty bands of the predictions in the following.

We start by looking at the cross section as a function of the inclusive jet multiplicity, figure 2. While the "pure" MC@NLO approach is able to describe the inclusive cross section at NLO in good agreement with data, it fails to provide a good description of the number of additional jets. This is to be expected, since the first jet is only described at leading-order accuracy, and any further jet even only in the parton-shower approximation. The MENLOPS method improves that prediction by including higher-multiplicity tree-level matrix elements, which can be seen to lead to a better agreement with data. It becomes obvious though that scale variations within those tree-level matrix elements lead to rather large uncertainties in all jet bins. This is significantly improved in the first two jet bins by the MEPs@NLO method, which has NLO accuracy in these observables and demonstrates the expected reduction in perturbative uncertainties. With the reduced uncertainty comes a near-perfect agreement with experimental data. For the higher jet multiplicities $N_{\text {jet }} \geq 3$ one recovers the tree-level picture with its larger uncertainties as expected.

The picture is very similar when requiring jets with either $p_{\perp}>20 \mathrm{GeV}$ or $p_{\perp}>$ $30 \mathrm{GeV}$, also for all other observables we have studied. We thus restrict the plots in the following to the $p_{\perp}>30 \mathrm{GeV}$ case.

In figure 3 the transverse momenta of jets in the different event categories of $W+\geq$ 1,2,3-jets are displayed. The inclusive statements from the previous paragraphs translate directly onto these differential distributions: When sensitive to the $W+1$ - or $W+2$-jet processes only, the perturbative uncertainties become significantly smaller in the MEPs@NLO method leading to a much better agreement with data.

A similar observation is made in figure 4 for the scalar sum of the transverse momenta of the lepton and jets and $E_{T}^{\text {miss }}$. The inclusiveness of this observable makes the hard tail susceptible to contributions from high jet multiplicities, which are only described at leading-order accuracy and thus cause a larger uncertainty band. In the low- $H_{T}$ region for $W+1$ - or $W+2$-jet events on the other hand one can see the reduced scale band in MEPS@NLO.

As a final comparison, the separation between the two leading jets is studied in the $\Delta R$ and $\Delta \phi$ variables. It is clear that the pure Mc@NLO approach can not be expected to give a good description of such angular correlations due to its parton shower approximation for the second jet. For the MePs@NLO method it is impressive to see how the reduced scale uncertainty leads to very good agreement with the data also in these observables.

Let us note that here we have analysed perturbative uncertainties stemming from the matrix element part of the calculation. While the inclusion of next-to leading order 

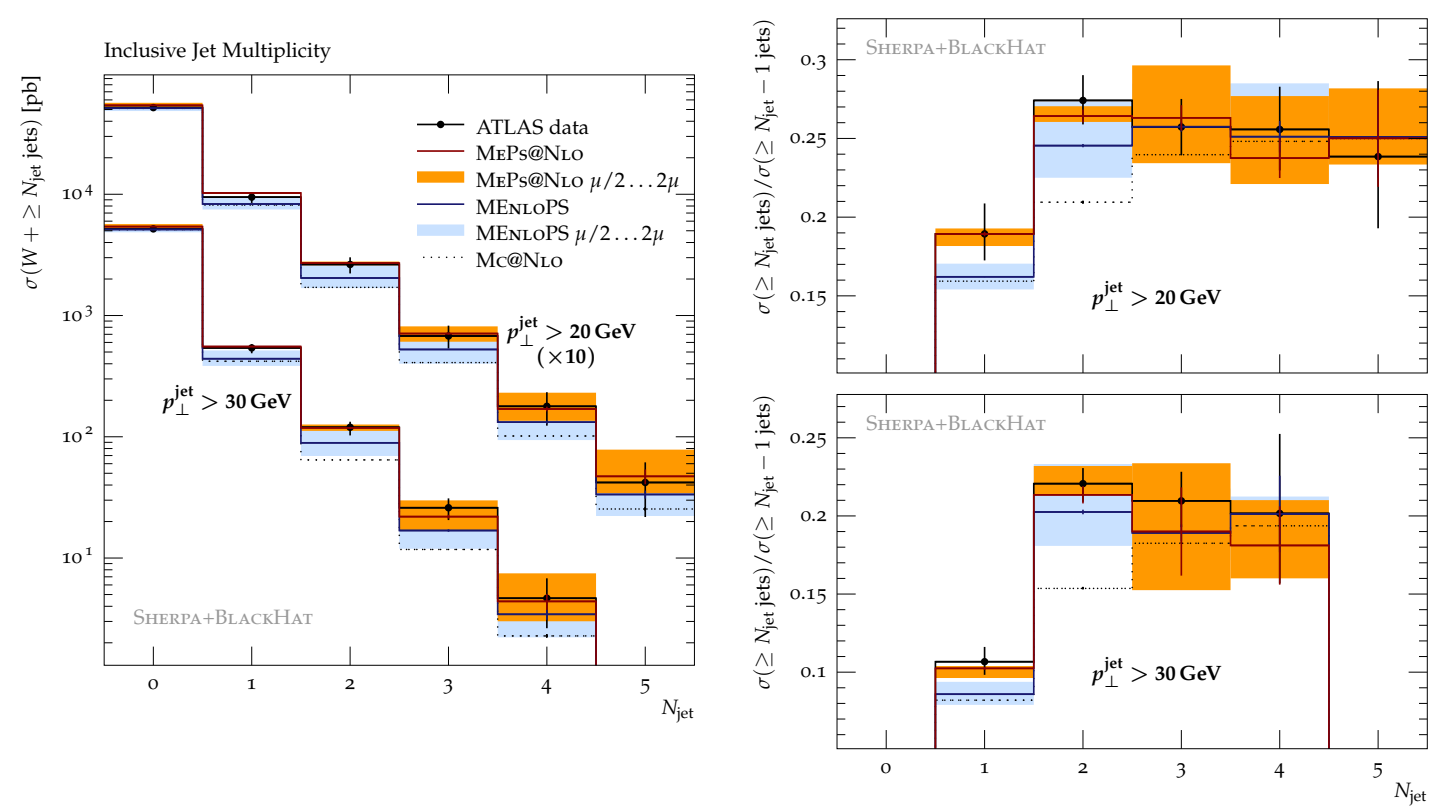

Figure 2. Cross section as a function of the inclusive jet multiplicity (left) and their ratios (right) in $W+$ jets events measured by ATLAS [58].

accuracy definitely reduces the related uncertainties, it is important to note that another source of theory ambiguities has not been discussed here. It is related to the effect of varying the resummation scale, here realised by the introduction of $\mu_{Q}$, in a way similar to the one performed in corresponding analytical calculations. It can be expected, though, that these uncertainties are fairly consistent between the MENLOPS approach and the new MEPs@NLO method presented here. While they certainly deserve further studies, we restricted our discussion here to the effect of theory uncertainties in the matrix element region, where we actually achieved a dramatic improvement over existing methods.

\section{Conclusions}

In this publication we have introduced a new method to consistently combine towers of matrix elements, at next-to leading order, with increasing jet multiplicity into one inclusive sample. Our method respects, at the same time, the fixed order accuracy of the matrix elements in their respective section of phase space and the logarithmic accuracy of the parton shower. The analysis of scale dependencies allows for a solid understanding of the corresponding theory uncertainties in the merged samples. Employing next-to leading order matrix elements leads, of course, to a dramatic reduction of the dependence on the renormalisation and factorisation scale and a much improved description of data. The same findings also apply to the case of $e^{-} e^{+}$annihilations into hadrons, cf. [43].

This allows, for the first time, to use Monte Carlo tools to generate inclusive multijet samples and analyse their uncertainty due to the truncation of the perturbative series in the matrix elements in a systematic and meaningful way. 

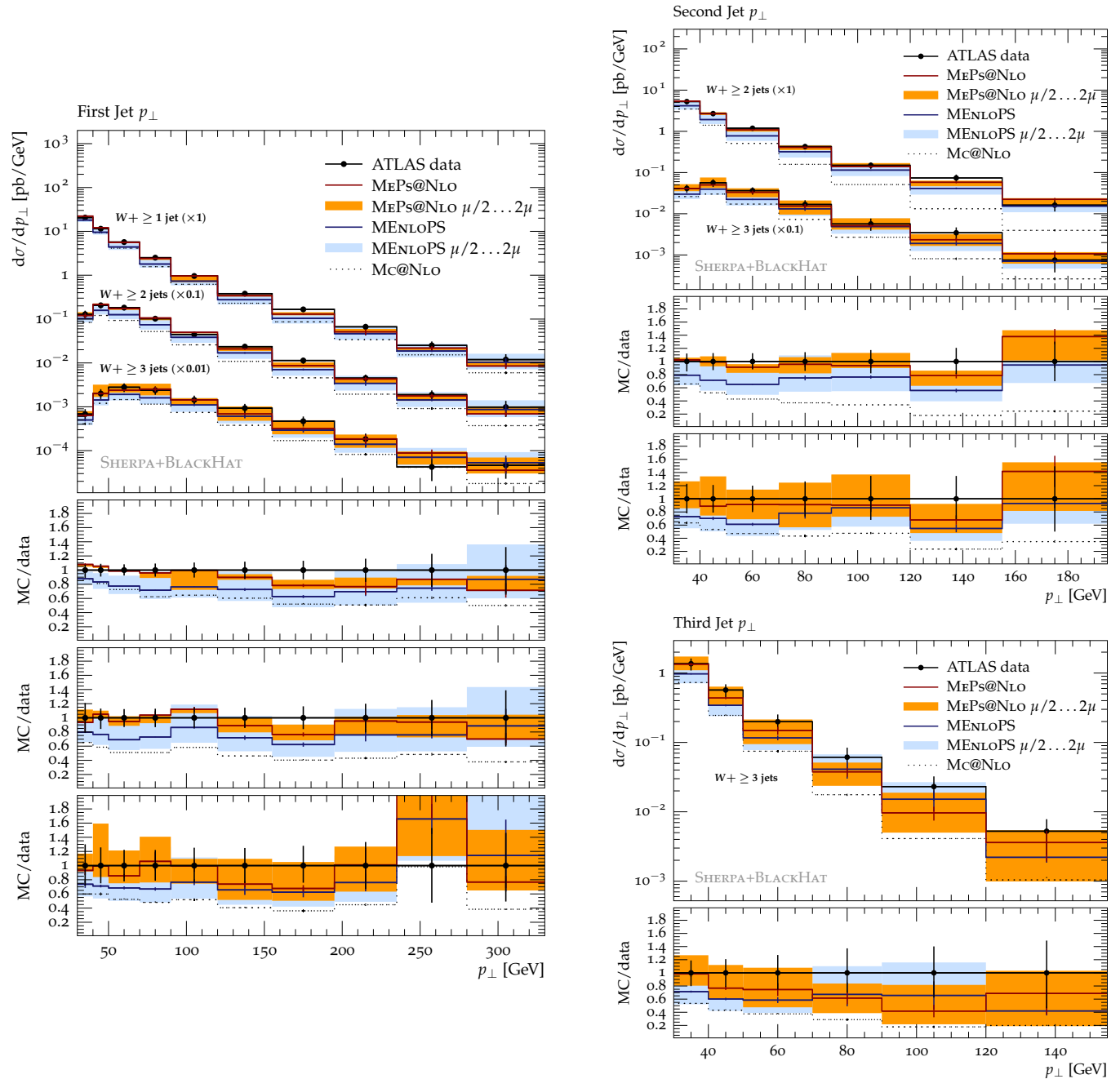

Figure 3. Differential cross section as a function of the transverse momentum of the first, second and third jet. All distributions are displayed for events selected to contain at least one, two or three jets.

\section{Acknowledgments}

SH's work was supported by the US Department of Energy under contract DE-AC0276SF00515, and in part by the US National Science Foundation, grant NSF-PHY-0705682, (The LHC Theory Initiative). MS's work was supported by the Research Executive Agency (REA) of the European Union under the Grant Agreement number PITN-GA-2010-264564 (LHCPhenoNet). FS's work was supported by the German Research Foundation (DFG) via grant DI 784/2-1. We gratefully thank the bwGRiD project for computational resources.

Open Access. This article is distributed under the terms of the Creative Commons Attribution License which permits any use, distribution and reproduction in any medium, provided the original author(s) and source are credited. 

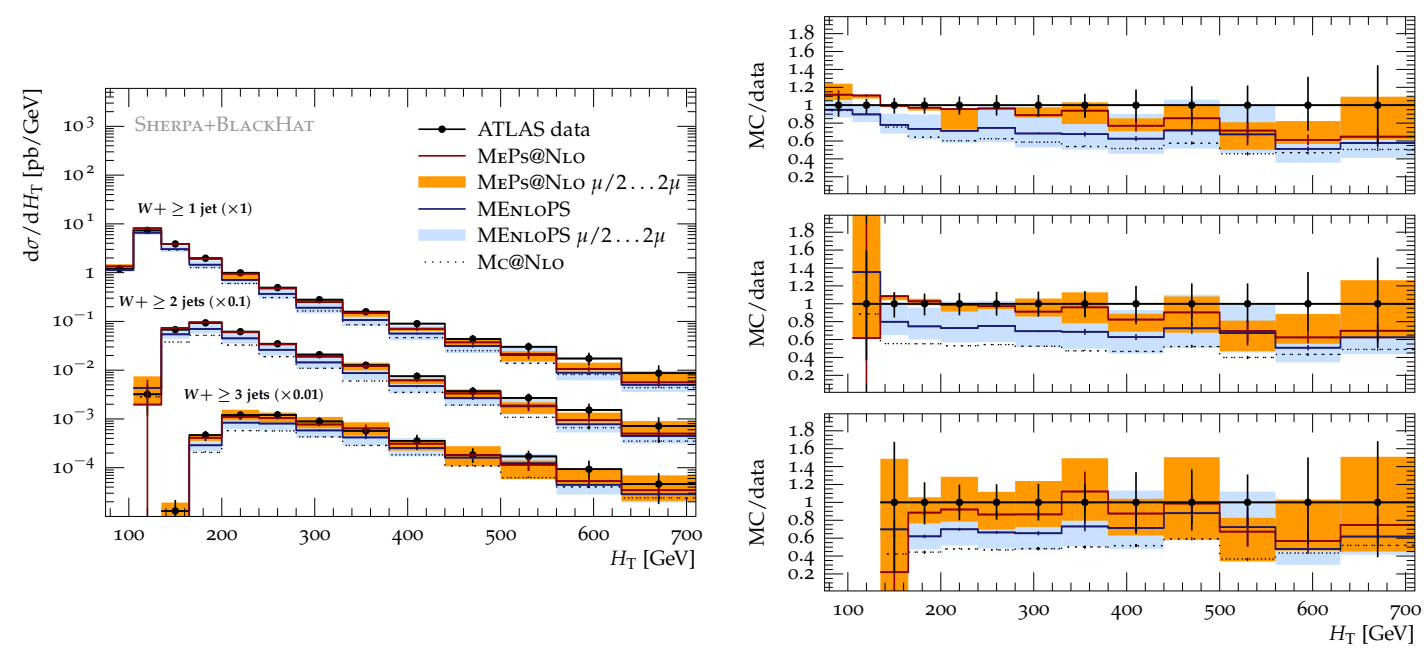

Figure 4. Differential cross section as a function of the scalar transverse momentum sum $H_{T}$ (left) and ratios to data from ATLAS [58] (right). Displayed are again contributions from events with at least one, two or three jets.
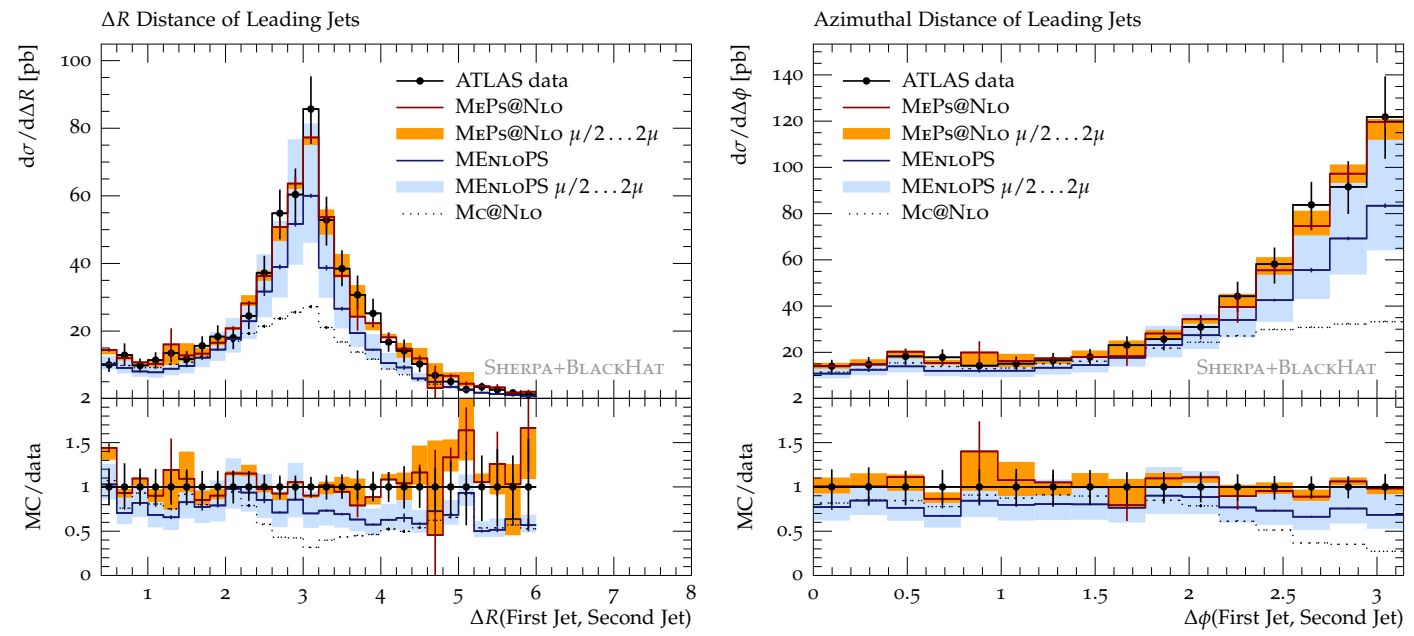

Figure 5. Differential cross section of $W+2$ jets events as a function of the jet separation in $R$ (left) and $\phi$ (right). The predictions are compared to experimental data from ATLAS [58].

\section{References}

[1] A. Denner and S. Dittmaier, Reduction schemes for one-loop tensor integrals, Nucl. Phys. B 734 (2006) 62 [hep-ph/0509141] [INSPIRE].

[2] G. Ossola, C.G. Papadopoulos and R. Pittau, Reducing full one-loop amplitudes to scalar integrals at the integrand level, Nucl. Phys. B 763 (2007) 147 [hep-ph/0609007] [INSPIRE].

[3] R.K. Ellis, W. Giele and Z. Kunszt, A numerical unitarity formalism for evaluating one-loop amplitudes, JHEP 03 (2008) 003 [arXiv:0708.2398] [INSPIRE].

[4] T. Binoth, J.-P. Guillet, G. Heinrich, E. Pilon and T. Reiter, Golem95: a numerical program to calculate one-loop tensor integrals with up to six external legs, Comput. Phys. Commun. 180 (2009) 2317 [arXiv:0810.0992] [INSPIRE]. 
[5] G. Ossola, C.G. Papadopoulos and R. Pittau, On the rational terms of the one-loop amplitudes, JHEP 05 (2008) 004 [arXiv:0802.1876] [INSPIRE].

[6] C. Berger et al., An automated implementation of on-shell methods for one-loop amplitudes, Phys. Rev. D 78 (2008) 036003 [arXiv:0803.4180] [inSPIRE].

[7] R.K. Ellis, W.T. Giele, Z. Kunszt and K. Melnikov, Masses, fermions and generalized D-dimensional unitarity, Nucl. Phys. B 822 (2009) 270 [arXiv:0806.3467] [INSPIRE].

[8] R.K. Ellis, K. Melnikov and G. Zanderighi, $W+3$ jet production at the Tevatron, Phys. Rev. D 80 (2009) 094002 [arXiv:0906.1445] [INSPIRE].

[9] C. Berger et al., Precise predictions for $W+4$ jet production at the Large Hadron Collider, Phys. Rev. Lett. 106 (2011) 092001 [arXiv: 1009.2338] [INSPIRE].

[10] H. Ita et al., Precise predictions for $Z+4$ jets at hadron colliders, Phys. Rev. D 85 (2012) 031501 [arXiv: 1108.2229] [inSPIRE].

[11] A. Denner, S. Dittmaier, S. Kallweit and S. Pozzorini, NLO QCD corrections to WWbb production at hadron colliders, Phys. Rev. Lett. 106 (2011) 052001 [arXiv:1012.3975] [INSPIRE].

[12] A. Bredenstein, A. Denner, S. Dittmaier and S. Pozzorini, NLO QCD corrections to top anti-top bottom anti-bottom production at the LHC: 2. Full hadronic results, JHEP 03 (2010) 021 [arXiv: 1001.4006] [INSPIRE].

[13] G. Cullen et al., Automated one-loop calculations with GoSam, Eur. Phys. J. C 72 (2012) 1889 [arXiv:1111.2034] [INSPIRE].

[14] G. Cullen et al., GoSam: a program for automated one-loop calculations, J. Phys. Conf. Ser. 368 (2012) 012056 [arXiv:1111.6534] [INSPIRE].

[15] F. Cascioli, P. Maierhofer and S. Pozzorini, Scattering amplitudes with open loops, Phys. Rev. Lett. 108 (2012) 111601 [arXiv:1111.5206] [INSPIRE].

[16] V. Hirschi et al., Automation of one-loop QCD corrections, JHEP 05 (2011) 044 [arXiv: 1103.0621] [INSPIRE].

[17] S. Frixione and B.R. Webber, Matching NLO QCD computations and parton shower simulations, JHEP 06 (2002) 029 [hep-ph/0204244] [INSPIRE].

[18] P. Torrielli and S. Frixione, Matching NLO QCD computations with PYTHIA using MC@NLO, JHEP 04 (2010) 110 [arXiv: 1002.4293] [INSPIRE].

[19] S. Frixione, F. Stoeckli, P. Torrielli and B.R. Webber, NLO QCD corrections in HERWIG++ with MC@NLO, JHEP 01 (2011) 053 [arXiv: 1010.0568] [INSPIRE].

[20] R. Frederix et al., aMC@NLO predictions for Wjj production at the Tevatron, JHEP 02 (2012) 048 [arXiv: 1110.5502] [INSPIRE].

[21] R. Frederix et al., Four-lepton production at hadron colliders: aMC@NLO predictions with theoretical uncertainties, JHEP 02 (2012) 099 [arXiv:1110.4738] [INSPIRE].

[22] S. Hoeche, F. Krauss, M. Schonherr and F. Siegert, $W+n$-jet predictions at the Large Hadron Collider at next-to-leading order matched with a parton shower, Phys. Rev. Lett. 110 (2013) 052001 [arXiv: 1201.5882] [INSPIRE].

[23] P. Nason, A New method for combining NLO QCD with shower Monte Carlo algorithms, JHEP 11 (2004) 040 [hep-ph/0409146] [INSPIRE].

[24] S. Frixione, P. Nason and C. Oleari, Matching NLO QCD computations with parton shower simulations: the POWHEG method, JHEP 11 (2007) 070 [arXiv: 0709.2092] [INSPIRE]. 
[25] K. Hamilton, P. Richardson and J. Tully, A positive-weight next-to-leading order Monte Carlo simulation for Higgs boson production, JHEP 04 (2009) 116 [arXiv: 0903.4345] [INSPIRE].

[26] S. Alioli, P. Nason, C. Oleari and E. Re, A general framework for implementing NLO calculations in shower Monte Carlo programs: the POWHEG BOX, JHEP 06 (2010) 043 [arXiv: 1002 . 2581] [INSPIRE].

[27] S. Hoche, F. Krauss, M. Schonherr and F. Siegert, Automating the POWHEG method in Sherpa, JHEP 04 (2011) 024 [arXiv: 1008.5399] [INSPIRE].

[28] J.M. Campbell et al., NLO Higgs boson production plus one and two jets using the POWHEG BOX, MadGraph 4 and MCFM, JHEP 07 (2012) 092 [arXiv:1202.5475] [INSPIRE].

[29] C. Bernaciak and D. Wackeroth, Combining NLO QCD and electroweak radiative corrections to $W$ boson production at hadron colliders in the POWHEG framework, Phys. Rev. D 85 (2012) 093003 [arXiv:1201.4804] [INSPIRE].

[30] L. Barze, G. Montagna, P. Nason, O. Nicrosini and F. Piccinini, Implementation of electroweak corrections in the POWHEG BOX: single W production, JHEP 04 (2012) 037 [arXiv: 1202.0465] [INSPIRE].

[31] S. Catani, F. Krauss, R. Kuhn and B.R. Webber, QCD matrix elements + parton showers, JHEP 11 (2001) 063 [hep-ph/0109231] [INSPIRE].

[32] L. Lönnblad, Correcting the color dipole cascade model with fixed order matrix elements, JHEP 05 (2002) 046 [hep-ph/0112284] [INSPIRE].

[33] M.L. Mangano, M. Moretti and R. Pittau, Multijet matrix elements and shower evolution in hadronic collisions: $W b \bar{b}+n$ jets as a case study, Nucl. Phys. B 632 (2002) 343 [hep-ph/0108069] [INSPIRE].

[34] F. Krauss, Matrix elements and parton showers in hadronic interactions, JHEP 08 (2002) 015 [hep-ph/0205283] [INSPIRE].

[35] S. Hoeche, F. Krauss, S. Schumann and F. Siegert, QCD matrix elements and truncated showers, JHEP 05 (2009) 053 [arXiv:0903.1219] [INSPIRE].

[36] K. Hamilton, P. Richardson and J. Tully, A modified CKKW matrix element merging approach to angular-ordered parton showers, JHEP 11 (2009) 038 [arXiv:0905.3072] [INSPIRE].

[37] L. Lönnblad and S. Prestel, Matching tree-level matrix elements with interleaved showers, JHEP 03 (2012) 019 [arXiv: 1109.4829] [INSPIRE].

[38] K. Hamilton and P. Nason, Improving NLO-parton shower matched simulations with higher order matrix elements, JHEP 06 (2010) 039 [arXiv: 1004.1764] [INSPIRE].

[39] S. Hoche, F. Krauss, M. Schonherr and F. Siegert, NLO matrix elements and truncated showers, JHEP 08 (2011) 123 [arXiv:1009.1127] [INSPIRE].

[40] S. Hoeche, F. Krauss, M. Schonherr and F. Siegert, A critical appraisal of $N L O+P S$ matching methods, JHEP 09 (2012) 049 [arXiv:1111.1220] [INSPIRE].

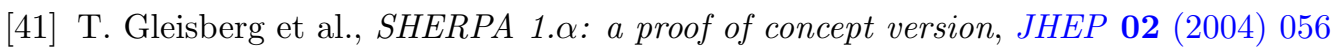
[hep-ph/0311263] [INSPIRE].

[42] T. Gleisberg et al., Event generation with SHERPA 1.1, JHEP 02 (2009) 007 [arXiv:0811.4622] [INSPIRE].

[43] T. Gehrmann, S. Hoche, F. Krauss, M. Schonherr and F. Siegert, NLO QCD matrix elements + parton showers in $e^{+} e^{-} \rightarrow$ hadrons, JHEP 01 (2013) 144 [arXiv:1207.5031] [INSPIRE]. 
[44] N. Lavesson and L. Lönnblad, $W+$ jets matrix elements and the dipole cascade, JHEP 07 (2005) 054 [hep-ph/0503293] [INSPIRE].

[45] T. Sjöstrand, A model for initial state parton showers, Phys. Lett. B 157 (1985) 321 [INSPIRE].

[46] R.K. Ellis, W.J. Stirling and B.R. Webber, QCD and collider physics, Cambridge Monogr. Part. Phys. Nucl. Phys. Cosmol. volume 8, Cambridge University Press, Cambridge U.K. (1996).

[47] S. Catani and M. Seymour, A general algorithm for calculating jet cross-sections in NLO QCD, Nucl. Phys. B 485 (1997) 291 [Erratum ibid. B 510 (1998) 503-504] [hep-ph/9605323] [INSPIRE].

[48] S. Catani, S. Dittmaier, M.H. Seymour and Z. Trócsányi, The dipole formalism for next-to-leading order QCD calculations with massive partons, Nucl. Phys. B 627 (2002) 189 [hep-ph/0201036] [INSPIRE].

[49] G. Marchesini and B. R. Webber, Monte Carlo simulation of general hard processes with coherent QCD radiation, Nucl. Phys. B 310 (1988) 461 [INSPIRE].

[50] S. Gieseke, P. Stephens and B. Webber, New formalism for QCD parton showers, JHEP 12 (2003) 045 [hep-ph/0310083] [INSPIRE].

[51] F. Krauss, R. Kuhn and G. Soff, AMEGIC ++ 1.0: a matrix element generator in $\mathrm{C}++$, JHEP 02 (2002) 044 [hep-ph/0109036] [INSPIRE].

[52] T. Gleisberg and S. Hoeche, Comix, a new matrix element generator, JHEP 12 (2008) 039 [arXiv: 0808.3674] [INSPIRE].

[53] T. Gleisberg and F. Krauss, Automating dipole subtraction for QCD NLO calculations, Eur. Phys. J. C 53 (2008) 501 [arXiv:0709.2881] [inSPIRE].

[54] T. Binoth et al., A proposal for a standard interface between Monte Carlo tools and one-loop programs, Comput. Phys. Commun. 181 (2010) 1612 [arXiv:1001.1307] [INSPIRE].

[55] C. Berger et al., Next-to-leading order QCD predictions for $W+3$-jet distributions at hadron colliders, Phys. Rev. D 80 (2009) 074036 [arXiv:0907.1984] [INSPIRE].

[56] C.F. Berger et al., Next-to-leading order QCD predictions for $Z, \gamma^{*}+3$-jet distributions at the Tevatron, Phys. Rev. D 82 (2010) 074002 [arXiv: 1004.1659] [INSPIRE].

[57] S. Schumann and F. Krauss, A parton shower algorithm based on Catani-Seymour dipole factorisation, JHEP 03 (2008) 038 [arXiv:0709.1027] [INSPIRE].

[58] ATLAS collaboration, Study of jets produced in association with a $W$ boson in pp collisions at $\sqrt{s}=7$ TeV with the ATLAS detector, Phys. Rev. D 85 (2012) 092002 [arXiv: 1201.1276] [INSPIRE].

[59] A. Buckley et al., Rivet user manual, arXiv:1003.0694 [INSPIRE].

[60] M. Cacciari, G.P. Salam and G. Soyez, The anti- $k_{t}$ jet clustering algorithm, JHEP 04 (2008) 063 [arXiv: 0802.1189] [inSPIRE].

[61] M. Cacciari, G.P. Salam and G. Soyez, FastJet user manual, Eur. Phys. J. C 72 (2012) 1896 [arXiv:1111.6097] [INSPIRE].

[62] H.-L. Lai et al., New parton distributions for collider physics, Phys. Rev. D 82 (2010) 074024 [arXiv: 1007.2241] [INSPIRE]. 